\title{
Current ideas on the origin of stripes.
}

\author{
J. Zaanen \\ Institute Lorentz for Theoretical Physics, Leiden University \\ P.O.B. 9506, 2300 RA Leiden, The Netherlands \\ (October 23, 2018; E-mail: jan@lorentz.leidenuniv.nl)
}

\begin{abstract}
It will be attempted to present a coherent view on the current ideas regarding the origin of the stripe instability. Special emphasis will be put on the problem of how to combine the microscopic pictures, leaning on spin-charge topological aspects, with the notion of frustrated phase separation.

64.60.-i, 71.27.+a, 74.72.-h, 75.10.-b
\end{abstract}

I have noticed that newcomers to the field of stripes [1] seem to percieve an atmosphere of theoretical controversy surrounding the issue of stripe microscopy. It is actually so that there is a sense of growing consensus on what is understood - self-evidently, much remains still in the dark. The confusion finds its origin in the history of the subject: stripes were several times (re)discovered theoretically over the past ten years from quite different physical perspectives. It took a little time to realize that these different pieces of physics in fact all matter.

I will present here a crude sketch of the main ideas, ordered according to my personal view on this subject. Stripes were discovered for the first time by Gunnarsson and me [2] as a generic classical instability of doped MottHubbard insulators, with classical in the sense of 'integrating out fermions around the classical saddlepoint' lingo for Hartree-Fock. Although still of relevance, subsequent developments made clear that naive Hartree-Fock does miss some essential pieces of cuprate physics. I refer in the first place to the highly non-trivial role played by quantum fluctuations (section I): the realization by Prelovsek and coworkers [3] that the stripe instability emerges entirely from the soup of quantum fluctuations, linking the phenomenon to spin-charge separation; the very recent discovery by White and Scalapino [4] that stripes can be made out of pairs of holes, instead of single holes, suggesting interesting relationships with superconductivity.

A theorem by Laughlin states that a complicated theory which is right looses on the short term from a simple theory which is wrong [5]. I percieve the statement that stripes exist because there are short range attractive interactions and long range repulsive interactions as a theory of the second kind. It is even so that it can be directly seen from the experiments that such a statement does not make sense. This is different from claiming that longer range interactions (neglected in Hubbard models) do not exist. The frustrated phase separation mechanism [6] hits full force at $x>1 / 8$ where the stripes likely become internally charge compressible (section II).

\section{STRIPES AS HOLONS IN TWO DIMENSIONS.}

A first set of ideas emerged from microscopic calculations. They have in common that stripes can be looked at as 2D generalizations of the topological excitations known from 1D physics. One can excercise the notion that the holons from one dimensions do survive in $2 \mathrm{D}$ as long as they bind into stripes [7]. However, this should be handled with care: as the remainder will make clear, stripes have to be an irreducible two dimensional phenomenon which cannot be simply thought of as $N$ times one dimensional physics. I actually like to dream that stripes are about nature teaching us how to properly generalize the mathematical theory of one dimensional physics to higher dimensions. This theory is yet to be discovered, and all we posses at the moment are some vague contours of the real thing.

The lesson to be drawn from the early mean-field calculations is that the problem of the doped Mott-Hubbard insulator is on the semi-classical level a close relative of the doped Peierls insulator. In the latter, the doped holes bind to topological defects (domain walls) of the density wave, forming the so-called Su-Schrieffer-Heeger solitons [8]. Although the doped Mott-Hubbard insulator is different (the antiferromagnet is itself made out of electrons and the order parameter is a vector), this soliton mechanism was shown to survive in this context [2, 10,11, with the longitudinal Néel order parameter component taking the role of the phonon field of the SSH problem. As was substantiated by Zaanen and Oleś [12], the stability of the charged domain walls is best understood by first considering the hole motions perpendicular to the walls. This is like a one dimensional problem, where the order parameter defect pulls out a 'mid-gap' state from the Hubbard bands which is occupied by the carrier. This corresponds with a hole bound to an Ising-like domain wall, in the sense that the staggered order parameter changes sign upon passing the localized charge, regardless its overall orientation ('classical holon'). In two dimensions, these holons can only survive when they are 'put on a row' to satisfy the topological requirements of a 2D Néel order parameter. Remarkably, the energetics of at least the filled wall (one hole/domain wall unit 
cell) is one dimensional-like in the sense that most of the energy is gained by the motions of the holes perpendicular to the wall. For instance, these Hartree-Fock walls are characterized by an extremely soft transversal dynamics: as long as the holons form a connected trajectory, the energies associated with shape deformations of the walls are barely detectable. In a sense, this is like ' $N$ times one dimensional physics'. However, it is a specialty of this particular type of stripe phase. The stripes of relevance to cuprates are half-filled (one hole per domain wall unit cells), and as will be explained further in the next section, in order to acquire special stability a gap has to develop in the mid-gap band itself [12, 13]. Since the physics of a system of particles on a line is not reducible to that of particles living on disconnected points, these half-filled stripes are truely $2 \mathrm{D}$ objects.

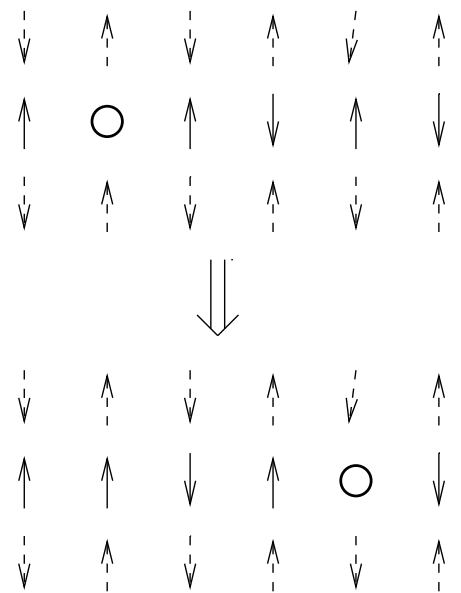

FIG. 1. In one dimension, charge-spin separation finds its origin in simple kinematics: a hole injected at the origin has transformed after a couple of hops into a domain wall-like spin defect (spinon) and a hole surrounded by antiparallel spins (holon). In two dimensions (dashed arrows), the spinon and holon are confined by the magnetic string potential: the spins are flipped along the trajectory connecting the spinon and the holon, and the number of parallel spins grows proportional with the distance between the two.

Despite their meat-and-potato image, classical saddlepoints are very serious objects. Since the static stripe phase is a classical vacuum (a Néel state and a charge density wave), there has to be a theory which is controlled by this vacuum, and this theory has to have the structure of Hartree-Fock: the quasiparticles can be integrated out by letting them scatter against the order parameter potential, and this zero-th order state can be adiabatically continued to the true vacuum. The caviat is of course that this theorem is only valid with regard to the appropriately renormalized Hamiltonian. There is no a-priori reason to believe that simple Hubbard models can claim this status. Nevertheless, it seems that Hubbard model Hartree-Fock gives a correct and even quantitatively meaningful description of the ground state of doped nickelates, where the stripes were first observed [14, 15].

That it also can go wrong is vividly illustrated by the more recent discovery of stripes in the $t-J$ model. It is easy to check that in terms of the bare spins and bare holes, there is no stripe instability in the classical $(S \rightarrow \infty)$ limit of the $t-J$ model. The $t-J$ model has to do with the large $U$ limit of the Hubbard model, and it was early on established [11] that stripes disappear on the mean-field level when $U$ becomes larger than twice the bandwidth. The stripes of the $t-J$ model are a genuine quantum order-out-of-disorder phenomenon. Without quantum fluctuations $(S \rightarrow \infty)$ stripes do not exist, and one has to go far beyond the Gaussian level (linear spin waves) to recover the stripe instability. In fact, Prelovsek and coworkers, who were the first to identify a tendency for stripe formation in the $t-J$ model [3], came up with a mechanism linking it to the holons of one dimensional physics.

In one dimensions, spin-charge separation is a trivial kinematical effect and it can be illustrated by a simple strong coupling cartoon [16. A bare hole can freely hop in a $S=1 / 2$ spin system, and after a couple of hops the hole has 'decayed' into a Ising-like domain 'wall' (better viewed as a Jordan-Wigner fermion; the spinon) while the hole is surrounded by anti-parallel spins: the hole is 'bound' to a domain wall (Fig. 1). Although this holon looks similar to the 'classical holon' of the Hartree-Fock solutions, it exists because of the delocalization of charge which is not present in the semiclassical theory. This kinematical effect is unavoidable in any clean one dimensional system and spin-charge separation is physical law. In two dimensions, the spinon and holon are connected by a 'magnetic string' of flipped spins (Fig. 1): the energy grows linearly with the spinon-holon separation because of the ferromagnetic bonds to the spins neighboring the string. At least in the well understood one hole case, the short time dynamics is spinon-holon like, but at low energies these confine to form a hole - the well known Landauesque quasiparticle [17].

Prelovsek and coworkers [3] recognized that in the many hole problem there is another possibility: holons do survive but they condense in connected trajectories corresponding with fluctuating stripes. The underlying mechanism adds a completely new meaning to the expression 'electron correlations'. Start out with a stripe, viewed as a string of holons (Fig. 2). What happens when an individual holon hops to a neighboring lattice site? The spin moves backward, but since the stripe is an antiphase boundary, this spin ends up in a 'right' spin domain, not causing any ferromagnetic bond. A neighboring holon can now move even more easily, and the net result is that two kinks propagate away freely, connected by a piece of stripe which is displaced by a lattice constant (Fig. 2) [18]. Because all holons can hop, these kinks will tend to proliferate, thereby delocalizing the stripe as 
a whole. Hence, although the price is paid of a reduced hopping configuration space, individual charges can hop as if there is no antiferromagnetic background hindering their motions, by coordinating these hoppings with those of all other particles. Prelovsek et al. substantiated this qualitative idea with extensive quantitative calculations [3]. Intrigued by these observations, we spend in Leiden much time on studying further abstracted 'lattice string' models. Given that kinks proliferate as just described, how does the string as a whole fluctuate? This turned out to be a rather amusing affair, with links to the hidden order of Haldane spin chains, surface statistical physics, etcetera. The tentative conclusion is a 'don't worry theorem': when these strings delocalize, their long wavelength dynamics is always of the simplest possible kind, namely the one governed by free field theory [19].

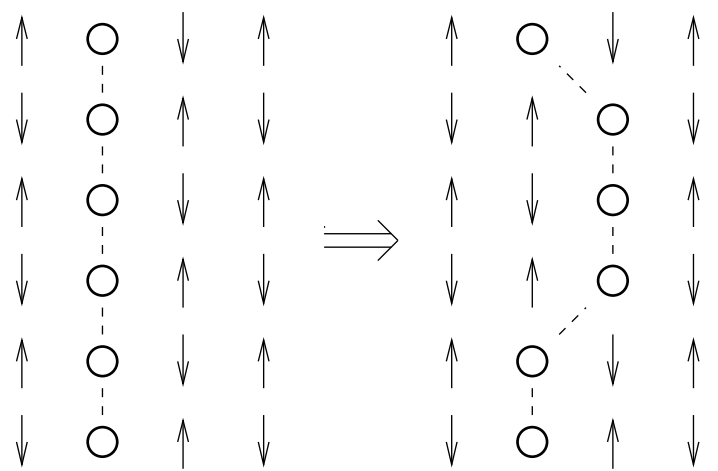

FIG. 2. The order-out-of-disorder mechanism of Prelovsek and Zotos. The magnetic string potential can be avoided in 2D by forming closed trajectories of holons: when an individual hole hops, the spin moves backwards into its right spin domain. The price is paid of a reduced hopping phase space, but much of the kinetic energy is recovered by the delocalization of the 'string' as a whole.

The exact diagonalization studies by White and Scalapino [4 show that the above story is still incomplete. Although the dust has not settled yet, their message appears to be that the stripes in $S=1 / 2$ systems are made out of pairs of charges instead of single charges [20]. This is appealing, if not only because it has to be explained how superconductivity enters the picture [21]. It is apparently so that in the considerations by Prelovsek et al the local quantum spin fluctuation is underestimated. These tend to stabilize local singlet spin pairs, especially so when holes are in their neighborhood. The simulations show that there is a tendency to form a bound state consisting of two holes and two spins. The holes tend to sit on opposite sides of the elementary plaquette, while the two spins on the other corners form a strong singlet bond along the diagonal of the plaquette. Leaving it to Scalapino et al. to explain this pairing mechanism in more detail, it is easy to see that they cause anti-phase Néel correlations in the spin background. The singlets are formed along the diagonal of the plaquette, forcing anti-parallel spin correlation on the same sublattice. If the surrounding spin system is near to its classical limit, the singlet-triplet logic of e.g. the bilayer Heisenberg model [22] applies and it follows that these pairs act like local antiphase magnetic boundaries ('bosonic holons').

\section{RELEVANCE OF LONG RANGE INTERACTIONS.}

As already announced in the introduction, there is a second set of ideas which do not fit in directly in the smooth flow of ideas alluded to in the previous section: frustrated phase separation [6]. There is little to be explained, since the beauty of the argument is in its simplicity: a classical system of particles on a lattice, characterized by short range attractions and long range repulsions, minimizes its energy by forming linear domains of enhanced and reduced density. In the context of stripes, it is sometimes argued that this is the big number physics and in the space left behind after all this has happened, subtleties can occur of the kind described in the previous section. The problem with this kind of argument is that it relies on a percieved detailed knowledge of the short wavelength physics, and it is a long time experience (at least mine) that this regime is littered with messy intricacies [23]. Instead, the tractable question is as to what extent long range interactions are consequential for the long wavelength physics. For instance, under the rule of frustrated phase separation, the Rome group [24] might well be on the right track developing the relevant field theory. Longitudinal fluctuations are expected to dominate: holes fluctuate from hole rich to hole poor regions thereby decreasing the amplitude of the stripe order parameter. Alternatively, when the 'holon glue' dominates, the stripes disorder by transversal (shape-changing) fluctuations and the string liquid ideas $13,19,25$, become more natural. The truth is of course somewhere in the middle, and it is a matter of high urgency to find out where this somewhere is.

Regardless the importance of long range interactions, it is not always realized that the 'holon-glue' of the previous section is also about big numbers. A key consequence of section I is that stripes are also anti-phase boundaries in the spin system - a fact not addressed at all by frustrated phase separation. Obviously, the strength of the exchange interaction $J^{\prime}$ connecting the spins on opposite sides of the domain wall is intimately related to the tendency of the hole (or pair) to surround itself with antiparallel spins and this exchange interaction is actually quite large. The spin waves have been measured in a nickelate stripe phase [26], indicating that $J^{\prime}$ is smaller by no more than a factor of two as compared to the exchange at half filling. In addition, the dynamical fluctuations in superconducting cuprates remain incommensurate up to rather large temperature- and frequencies [27], and this is 
only possible when $J^{\prime}$ is at least as large. The implication is that a lower bound to the strength of the 'holon-glue' interaction is actually known and this quantity is of order of the exchange interaction at half-filling - a big number.

As emphasized by Emery and Kivelson 28, the behavior of the stripe period as function of doping gives away an important clue. It was already emphasized that, since static stripes do form a classical vacuum, a theory with the structure of Hartree-Fock should exist. It is possible to make deductions based merely on the structure of the theory: if the effective Hamiltonian is characterized by only short range interactions, special stability is only obtained if the order parameter potential is such that it causes a gap in the electronic spectrum in at least part of the Brillioun zone. Applied to stripes, the mere magnitude of the spins in the magnetic domains $\left(>0.3 \mu_{B}\right.$ at $x=1 / 8$ implies that a rather large gap is associated with the electrons moving in these domains. The quasi $1 \mathrm{D}$ electronic subsystem living on the wall is more delicate. If the instability lives in the diagonal (spin density and/or charge density) channel, gaps can only occur when the charge density is commensurate with the underlying lattice. For instance, starting from Hubbard models one finds a preferred density of one hole per domain wall unit cell (filling fraction $\nu=1$ ) corresponding with a gap between the completely empty mid-gap band and the lower Hubbard band. Alternatively, special stability at $\nu=1 / 2$ (one hole per two unit cells) implies the presence of either a $2 k_{F}$ [12] or $4 k_{F}$ [13] density wave instability on the stripes.

If on-stripe charge-commensuration dominates, every hole adds a fixed length of domain wall and it follows directly that the domain wall spacing $d$ is inversely proportional to the number of holes $x$, while the incommensurability $\varepsilon \sim 1 / d \sim x$. In the nickelates this rule is very closely obeyed at low temperatures [29] and since $\nu=1$ Hubbard model Hartree-Fock appears as a sensible theory [15]. The situation in the cuprates is more interesting [1]. For doping levels $x<1 / 8$, one finds again $\varepsilon \sim x$ with $\nu=1 / 2$, indicating on-stripe charge commensurability. However, for $x>1 / 8$ the stripe period remains roughly constant, indicating that the stripes become internally charge compressible.

The significance of the frustrated phase separation argument is that it shows that long range interactions can change the basic rules of the game. In the presence of these interactions, charge density waves can acquire special stability, regardless the response of the states at the Fermi level. Charge density waves can exist in purely classical systems (e.g., the Wigner crystal). Consider the interesting situation of competing short range attractiveand long range repulsive interactions where, as a subtle compromise, the charges pile up in linear stripe-like domains. What happens when this system is mildly quantized? Since the kinetic energy favors a homogeneous state, the charges spread out thereby decreasing the mag- nitude of the charge modulation and likely also changing the ordering wavevector. However, it will do so in some smooth way, unrelated to the wavevectors spanning the emerging Fermi-surface. The system becomes a metal with a charge density modulation which is to zero-th order driven by the interactions alone.

The microscopic picture of the previous section and the principle exposed in the previous paragraph refer to completely different aspects of the physics, and there is nothing forbidding that they are both active at the same time. Consider the classical limit but take instead of featureless local attractions a minimal way of incorporating the holon idea,

$$
\begin{aligned}
H= & \sum_{\vec{i} \vec{\delta}} n_{\vec{i}} n_{\vec{i}+\vec{\delta}} \vec{M}_{\vec{i}} \cdot \vec{M}_{\vec{i}+\vec{\delta}}+ \\
& J_{h} \sum_{\vec{i} \vec{\delta}}\left(1-n_{\vec{i}}\right) n_{\vec{i}-\vec{\delta}} n_{\vec{i}+\vec{\delta}} \vec{M}_{\vec{i}-\vec{\delta}} \cdot \vec{M}_{\vec{i}+\vec{\delta}} .
\end{aligned}
$$

$1-n_{\vec{i}}$ is the number operator of the charged particle (the hole, or the pair of White and Scalapino) on the $\vec{i}$ site of the lattice while $\vec{M}$ denotes the direction of the staggered order parameter. This describes a classical Heisenberg magnet doped by 'classical holons': for $J_{h}>0$, individual charges want to be coordinated with an antiparallel configuration of the staggered magnetization. For the present argument it suffices to know that the ground state for $J_{h} \geq 1$ is a charge commensurate but transversally disordered stripe state, while for smaller $J_{h}$ phase separation occurs (Eq. (11) defines a surprisingly complicated statistical physics [30]). If long range charge-charge interaction is added, stripe long range order is stabilized and for $J_{h}=0$ these stripes are precisely of the frustrated phase separation kind. The 'holon interaction' disfavors broad stripes and when $J_{h}$ becomes larger the narrow 'holons on a row' stripes recover. However, these stripes are now 'doped' in the sense that additional holons are incorporated in the form of transversal kink defects carrying a net charge [30]. The mechanism is straightforwardly understood: in the frustrated phase separation limit $\left(J_{h}=0\right)$ the stripe period is set by a different mechanism (competition short- and long range interactions) than in the $J_{h} \rightarrow \infty$ limit (charge commensuration) and doped stripes appear as a compromise in the intermediate regime.

\section{AN OPEN ENDED STORY.}

There are reasons to believe that the understanding of even the 'easy' static stripes in the cuprates is still highly incomplete. Let me list some of the most obvious problems: (i) Why is there are a kink in $\varepsilon$ versus $x$ curve at $1 / 8$ [1]? It is not easy to see why this should happen, given the wisdom of the previous section: there is no obvious reason why the system should 
switch suddenly from on-stripe charge commensuration $(x<1 / 8)$ to stripe-to-stripe commensuration $(x>1 / 8)$. (ii) Why is it that the resistivity increases only slowly (like a logarithm) below the charge-ordering temperature? Stronger, why is the low temperature resistivity smallest at $x=1 / 8$, while it increases for both higherand lower dopings [31]? $x=1 / 8$ is the point of maximal commensuration and this is not at all reflected in transport properties. $\varepsilon \sim x$ should relate to the presence of some gap in the electronic spectrum and one would expect a strong asymmetry in the transport properties around $x=1 / 8$. This asymmetry is absent. (iii) How to incorporate properly superconductivity? It seems that the superconducting and stripe phases are connected by a (near) second order phase boundary, and it might even be that a coexistence ('antiferromagnetic supersolid') phase exists [1]. On the most general level, the dynamical stripe correlations showing up in the superconductors should be understood as reflecting this second order behavior: on 'short' (in fact, relatively large) length scales the system still remembers that it could become a stripe phase. Conversely, it should be the case that the stripe phase is also a superconductor, which failed at the very last moment. This raises some problems of principle. Focussing on the charge sector, the stripe phase is best called a complex solid, and the quantum liquid crystal ideas of Kivelson et al. 32 effectively illustrate what this can mean for the phase dynamics. The stripe phase is also an antiferromagnet. Although it is unclear to me why one should worry about a $0.6 \mu_{B}$ antiferromagnet at $x=0$, knowing about the lingering $0.3 \mu_{B}$ antiferromagnet at $x=0.15$, Zhang's $S O(5)$ ideas 33 make clear that there is still much to be learned concerning the problem of the nearcoexistence of an antiferromagnet and a superconductor. What is needed is an in-depth experimental characterization of the stripe phase.

Acknowledgements. Support is acknowledged by the Dutch Academy of Sciences (KNAW).

[1] J. M. Tranquada, Physica B, in press (condmat/9709325 and ref.'s therein.

[2] J. Zaanen and O. Gunnarsson, Phys. Rev. B 40, 7391 (1989).

[3] P. Prelovšek and X. Zotos, Phys. Rev. B 47, 5984 (1993); P. Prelovšek and I. Sega, Phys. Rev. B 49, 15241 (1994).

[4] S. R. White and D. J. Scalapino, cond-mat/9705128.

[5] R. J. Laughlin, unpublished.

[6] U. Löw et. al., Phys. Rev. Lett. 72, 1918 (1994).

[7] C. Nayak and F. Wilczek, Int. J. Mod. Phys. B 10, 2125 (1996)

[8] W. P. Su, J. R. Schrieffer, and A. J. Heeger, Phys. Rev.
B 22, 2099 (1980).

[9] P. Prelovšek and X. Zotos, Phys. Rev. B 47, 5984 (1993); P. Prelovšek and I. Sega, Phys. Rev. B 49, 15241 (1994).

[10] D. Poilblanc and T. M. Rice, Phys. Rev. B 39, 9749 (1989); H. J. Schulz, Phys. Rev. Lett. 64, 1445 (1990); M. Kato, K. Machida, H. Nakanitshi, and M. Fujita, J. Phys. Soc. Jpn. 59, 1047 (1990); J. A. Vergés, F. Guinea and E. Louis, Phys. Rev. B 46, 3562 (1992).

[11] M. Inui and P. B. Littlewood, Phys. Rev. B 44, 4415 (1991).

[12] J. Zaanen and A. M. Oles, Ann. Physik 5, 224 (1996).

[13] C. Nayak and F. Wilczek, Phys. Rev. Lett. 78, 2465 (1997).

[14] V. Sachan et. al., Phys. Rev. B 54, 12318 (1996).

[15] J. Zaanen and P. B. Littlewood, Phys. Rev. B 50, 7222 (1994).

[16] e. g., H. J. Schulz, in Correlated Electron Systems, ed. V. J. Emery (World Scientific, Singapore, 1993).

[17] E. Dagotto, Rev. Mod. Phys. 66, 763 (1994) and ref.'s therein.

[18] See also H. Viertiö and T. M. Rice, J. Phys. Cond. Matter 6, 7091 (1994).

[19] H. Eskes et. al., Phys. Rev. B 54, R724 (1996); ibid., unpublished.

[20] This possibility was for the first time pointed out in: H. Tsunetsugu, M. Troyer, and T. M. Rice, Phys. Rev. B 51, 16456 (1994).

[21] See also O. Zachar, S. A. Kivelson and V. I. Emery, in press; Yu. A. Krotov, D.-H. Lee and A. V. Balatsky, cond-mat/9705031.

[22] C. N. A. van Duin and J. Zaanen, Phys. Rev. Lett. 78, 3019 (1997).

[23] For instance, why is it so that $S r$ impurities only very weakly pin stripes, although calculations using $1 / \epsilon r$ potentials predict impurity potentials of order $0.5 \mathrm{eV}$ ? See V. I. Anisimov et al., Phys. Rev. Lett. 68, 345 (1992).

[24] C. Castellani, C. Di Castro and M. Grilli, condmat/9702112

[25] J. Zaanen, M. Horbach and W. van Saarloos, Phys. Rev. B 53, 8671 (1996).

[26] J. M. Tranquada, P. Wochner and D. J. Buttrey, Phys. Rev. Lett. 79, 2133 (1997).

[27] T. E. Mason et al., Phys. Rev. Lett. 68, 1414 (1992).

[28] V. J. Emery and S. A. Kivelson, this proceedings.

[29] A caviat is that this rule is violated at higher temperatures (P. Wochner et al., cond-mat/9706261). However, it should be realized that the range of validity of the above arguments is strictly limited to zero temperature. For instance, although there is at least in the $x=1 / 3$ system a large gap in the electronic spectrum at low temperatures, this gap apparently closes at the charge ordering temperature (T. Katsufuji et al., Phys. Rev. B 54, R14230 (1996)), indicating that thermal fluctuations do play a highly interesting role.

[30] C. Lubritto, K. Pijnenburg, and J. Zaanen, unpublished.

[31] B. Büchner et al., Phys. Rev. Lett. 73, 1841 (1994).

[32] S. A. Kivelson, E. Fradkin and V. J. Emery, condmat/9707327.

[33] S. C. Zhang, Science 257, 4126 (1997); this proceedings. 